\title{
All Carbon-Linked Continuous Three-Dimensional Porous Aromatic Framework Films with Nanometer-Precise Controllable Thickness
}

Martin Ratsch ${ }^{1}$, Chen Ye ${ }^{1}$, Yizhou Yang ${ }^{1}$, Airui Zhang ${ }^{2}$, Austin M. Evans ${ }^{3}$, Karl Börjesson ${ }^{\text {1* }}$

\footnotetext{
${ }^{1}$ University of Gothenburg, Department of Chemistry and Molecular Biology, Kemigården 4, 41296 Gothenburg, Sweden

${ }^{2}$ Division of Energy and Environmental Measurement, National Institute of Metrology, China NIM, Beijing, 100013, P. R. China

${ }^{3}$ Department of Chemistry, Northwestern University, Evanston, IL, 60208 USA
} 


\section{Table of Contents}

Materials and methods

Building block synthesis

S6

Supplementary Figures

S8

References

S14

Ratsch, Ye, Yang, Zhang, Evans and Börjesson - Supplementary Material - S2 


\section{Materials and methods}

General: All starting materials were purchased from Sigma-Aldrich Chemical Co. and used without further purification. All moisture and oxygen-sensitive reactions were carried out using Schlenk techniques in oven-dried glassware or in a nitrogen atmosphere glovebox. Solvents used for moisture and oxygen-sensitive reactions were dried using an MBraun MB SPS-800 solvent purification system. Flash chromatography was performed by a Teledyn Combiflash EZ prep using liquid chromatography mass spectrometry (LC-MS) grade solvents and normal phase silica with mesh size 230-400, particle size 40-63 micron, and pore size $60 \AA .{ }^{1} \mathrm{H}$ NMR spectra were recorded on a Varian spectrometer at $400 \mathrm{MHz}$. $J$-coupling values are given in Hertz $(\mathrm{Hz})$ and chemical shifts are given in ppm using tetramethysilane (TMS; $\delta=0.00 \mathrm{ppm}$ ) as an internal standard. The building block (tetrakis(4bromophenyl)methane, TBPM) was synthesized in two steps starting from chlorotriphenylmethane, following a literature procedure ${ }^{1}$ that is described in detail in the supporting information.

PAF-1 film synthesis: A quartz crystal microbalance (QCM) Q-Sense E4 Quartz Crystal Microbalance (QCM-D / Q-Sense Analyzer) from Biolin Scientific (with Kalrez ${ }^{\circledR}$ O-rings and gaskets) was used. QCM gold sensors with $\mathrm{Cr}$ or Ti adhesive layers were purchased from Biolin Scientific. The gold sensors were cleaned with a UV/ozone treatment for two hours and were then immersed in an $80^{\circ} \mathrm{C}$ 5:1:1 solution of milliQ (mQ)-water, ammonia (32\%), and hydrogen peroxide (30\%) for $45 \mathrm{~min}$. Next, the cleaned gold chips were washed with mQ-water and $\mathrm{EtOH}$, dried under a $\mathrm{N}_{2}$ stream, and stored for 1-4 days in a 24-well cell culture plate in a solution of 4-bromothiophenol (1 mM in EtOH). Before film synthesis, the coated sensor was taken out, rinsed with $\mathrm{EtOH}$, blow dried under a stream of $\mathrm{N}_{2}$, and placed into the QCM chamber. When the temperature was stable at $25^{\circ} \mathrm{C}$, the whole set-up was flushed with the solvent mixture (THF:DMF, 1:1) until all air bubbles were pushed out. Three programmable Legato110 OEM syringe pumps from KD Scientific were connected to a computer and controlled using Adagio 1.0 software. The first pump was charged with a solution of the building block (TBPM, $1.3 \mathrm{mg}$, $2 \mu \mathrm{mol}$, in $24 \mathrm{ml}$ THF:DMF; 1:1) and the second pump was charged with a solution of the coupling reagents 2,2'-bipyridine (1.7 mg, $10.9 \mu \mathrm{mol}, 4.9$ equiv.), bis(1,5-cyclooctadiene)nickel(0) $(3.1 \mathrm{mg}$, $11.3 \mu \mathrm{mol}, 5.12$ equiv.) and cis,cis-1,5-cyclooctadiene (1.35 $\mu 1,11.1 \mu \mathrm{mol}, 5.04$ equiv.) in $24 \mathrm{ml}$ 
THF:DMF 1:1. The third pump contained only the solvent mixture (THF:DMF 1:1, $24 \mathrm{ml}$ ). The syringe pumps pushed the fluids from syringe one and two (flow speed: $1 \mathrm{ml} \mathrm{hr}^{-1}$ each) through the system for $12-22 \mathrm{hr}$ while the resonance frequency shift was constantly measured and the $11^{\text {th }}$ overtone was used for further analysis (see Figure S6 for a comparison between overtones). In the end, the two syringe pumps switched off and the tubing and the chamber was washed for 1-3 hours using the third syringe pump $\left(1 \mathrm{ml} \mathrm{hr}^{-1}\right)$. After pushing air through the system, the sensor was removed and dried under a stream of nitrogen.

BCMP-2 film synthesis: A QCM Q-Sense E4 Quartz Crystal Microbalance (QCM-D / Q-Sense Analyzer) from Biolin Scientific (with Kalrez ${ }^{\circledR}$ O-rings and gaskets) was used. QCM gold sensors with $\mathrm{Cr}$ adhesive layers were purchased from Biolin Scientific. The gold sensors were cleaned with a UV/ozone treatment for two hours and afterwards immersed in an $80^{\circ} \mathrm{C}$ hot 5:1:1 solution of mQ-water, ammonia (32\%), and hydrogen peroxide (30\%) for $45 \mathrm{~min}$. Next, the cleaned gold chips were washed with mQ-water and EtOH, dried under a $\mathrm{N}_{2}$ stream, and stored for 1-4 days in a 24-well cell culture plate in a solution of 4-bromothiophenol (1 mM in EtOH). Before film synthesis, the coated sensor was taken out, rinsed with $\mathrm{EtOH}$, blow dried under a stream of $\mathrm{N}_{2}$, and placed into the QCM chamber. When the temperature was stable at $25^{\circ} \mathrm{C}$, the whole set-up was flushed with the solvent mixture (DMF:DIPA, 1:1) until all air bubbles were pushed out. Three programmable Legato110 OEM syringe pumps from KD Scientific were connected to a computer and controlled using Adagio 1.0 software. The first pump was charged with a solution of the building blocks (TBPM, $1.6 \mathrm{mg}, 2.5 \mu \mathrm{mol}$; 1,4-diethynylbenzene 1.0 $\mathrm{mg}, 7.9 \mu \mathrm{mol}$; in $24 \mathrm{~mL}$ DMF:DIPA 1:1) and the second pump was charged with a solution of catalysts tetrakis(triphenylphosphine)palladium(0) $(2 \mathrm{mg}, 1.7 \mu \mathrm{mol}, 0.7$ equiv.) and $\mathrm{CuI}(1 \mathrm{mg}, 5.3 \mu \mathrm{mol}, 2.1$ equiv.) in 24mL DMF:DIPA 1:1. The third pump contained only the solvent mixture (DMF:DIPA 1:1, $24 \mathrm{ml}$ ). The syringe pumps pushed the fluids from syringe one and two (flow speed: $1 \mathrm{ml} \mathrm{hr}^{-1}$ each) through the system for 12-22 hr while the resonance frequency shift was constantly measured and the $11^{\text {th }}$ overtone was used for further analysis. In the end, the two syringe pumps switched off and the tubing and the chamber was washed for 1-3 hours using the third syringe pump (1 $\left.\mathrm{ml} \mathrm{hr}^{-1}\right)$. After pushing air through the system, the sensor was removed and dried under a stream of nitrogen. 
PAF films analysis: Before observation, the chips were cleaned from potential dust using a stream of nitrogen. A Zeiss Axio Scope A1 and a Zeiss Axioscope 5 were used to analyze the macroscopic distribution of material on the QCM chips. Scanning electron microscopy (SEM) and Energy dispersive x-ray spectroscopy-scanning electron microscopy (EDX-SEM) were performed using a Quanta200 ESEM or a JEOL JSM-7800F Prime and a LEO Ultra 55 electron microscope, respectively. The microscopes (spatial resolution: $<2 \mathrm{~nm}$ ) were used with an acceleration voltage of $3-6 \mathrm{keV}$ to avoid charging effects on the surface. To assess the cross section of films, the QCM chips were broken, cleaned from dust and potential small pieces of the cross section with a nitrogen stream, and placed endways in the sample holder. To break the chips, they were held in between two tweezers, and by increasing the tension on both sides, breakage occurred. To gain evidence that the SAM formation occurred, the water contact angle was measured 10 times on the templated gold surface, resulting in angles consistently between 90 and 99 degrees (average 95 degrees). The values are thus much larger than those for clean gold (60-65 degrees according to the literature $)^{2}$, confirming the formation of the SAM. X-ray photoelectron spectroscopy (XPS) analysis was performed using a Thermo Scientific ESCALAB XI+ ${ }^{+}$spectrometer with an Al K-Alpha source. Etching of the surface was performed with a Monatomic and Gas Cluster Ion Source (MAGCIS) accessory at $200 \mathrm{eV}$. AFM was performed using an NTEGRA AFM from NT-MDT Spectrum instruments. Water contact angle measurements were performed with a Theta OneAttension from Biolin Scientific. X-ray reflectivity measurements were collected on a Rigaku Smartlab using a Bragg-Brentano geometry with an X-ray wavelength of $1.54 \AA$. Electron density profiles were fit using the REFLEX toolbox. ${ }^{3}$ Fitting parameters were obtained by estimating roughness and thickness from SEM and AFM measurements and an iterative assignment of the electron density of the PAF Film. We attribute poor fitting in the high-q regime to substantial roughness at buried interfaces which precluded more precise intensity fits. 


\section{Building block synthesis}

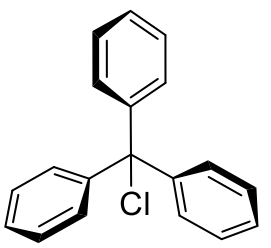

3. $\mathrm{H}_{3} \mathrm{PO}_{2}$

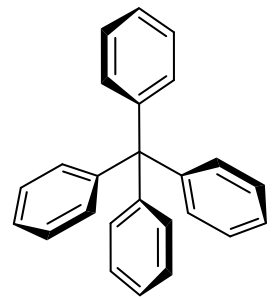

2

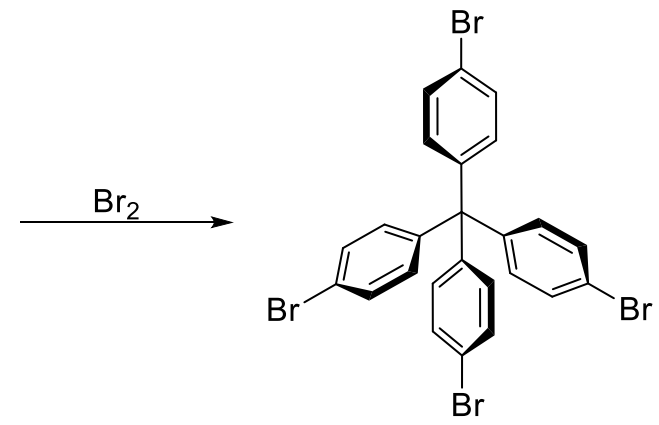

TBPM

Scheme S1. Synthetic procedure of the building blocks.

Synthesis of Tetraphenylmethane (2): A round bottomed flask was charged with chlorotriphenylmethane (1, $5.0 \mathrm{~g}, 17.9 \mathrm{mmol}, 1.00$ equiv.) and aniline (4.4 ml, $48.2 \mathrm{mmol}, 2.69$ equiv.). The reaction mixture was slowly heated to $190{ }^{\circ} \mathrm{C}$ under vigorous stirring. After 15 min, the mixture was cooled down to room temperature and a solution of aqueous $\mathrm{HCl}(2 \mathrm{M}, 20 \mathrm{~mL})$ and methanol (30 ml) were added to the flask. Afterwards the reaction mixture was heated to $80{ }^{\circ} \mathrm{C}$. After stirring for $30 \mathrm{~min}$ it was cooled down to room temperature and the resulting solid was filtered, washed with water $(100 \mathrm{~mL})$ and dried under vacuum. The dry solid was suspended in DMF $(50 \mathrm{~mL})$ and cooled to $-15{ }^{\circ} \mathrm{C}$. At this temperature, conc. sulfuric acid $(96 \%, 5.5 \mathrm{~mL})$ and isoamyl nitrite $(4 \mathrm{~mL}, 3.46 \mathrm{~g}, 29.9 \mathrm{mmol}$, 1.67 equiv.) were slowly added and the reaction mixture were left to stir for $1 \mathrm{hr}$. Afterwards hypophosphorous acid $(30 \%, 15 \mathrm{~mL})$ was added dropwise. When the addition was completed, the reaction mixture was heated to $50 \mathrm{C}$ and stirred until the evolution of gas ceased. Then, water $(15 \mathrm{ml})$ was added to precipitate the product and the suspension was filtered under suction and washed with water $(15 \mathrm{ml})$. After drying in vacuo $\left(70{ }^{\circ} \mathrm{C}\right.$, overnight $)$, tetraphenylmethane (2) was obtained as a bright brown powder $(5.45 \mathrm{~g}, 17.0 \mathrm{mmol}, 95 \%)$. The proton NMR data matched with literature values. ${ }^{1}$

${ }^{1} \mathrm{H}$ NMR $\left(400 \mathrm{MHz}, \mathrm{CDCl}_{3}\right.$, r.t. $): \delta(\mathrm{ppm})=7.28-7.16(\mathrm{~m}, 20 \mathrm{H}, \mathrm{ArH})$

Synthesis of tetrakis(4-bromophenyl)methane (TBPM): A round bottom flask was charged with bromine ( $1.65 \mathrm{ml}, 32.2 \mathrm{mmol}, 20.6$ equiv). At room temperature, tetraphenylmethane $(2,500 \mathrm{mg}$, $1.6 \mathrm{mmol}, 1.00$ equiv) was added in small portions. The mixture was stirred at room temperature for 30 
min and then cooled to $-78{ }^{\circ} \mathrm{C}$ and ethanol was added dropwise $(4 \mathrm{ml})$. After the addition was completed, the mixture was allowed to reach room temperature again in the open flask. The formed solid was filtered under vacuum and washed with aqueous sodium thiosulfate $(0.5 \mathrm{M}, 10 \mathrm{ml})$ and water $(10 \mathrm{ml})$ to yield a brown-yellowish solid that was thoroughly dried. Residual water was removed via dissolution of the solid in THF and subsequent addition of sodium sulfate. The crude product was dried under vacuum overnight. The product (TBPM) was purified via column flash chromatography ( $n$-pentane) and isolated as a yellowish powder $(327 \mathrm{mg}, 0.51 \mathrm{mmol}, 33 \%)$. The proton NMR data matched with literature values. ${ }^{1}$

${ }^{1} \mathrm{H}$ NMR $\left(400 \mathrm{MHz}, \mathrm{CDCl}_{3}\right.$, r.t.): $\delta(\mathrm{ppm})=7.39(\mathrm{dd}, J=8.6,1.4 \mathrm{~Hz}, 8 \mathrm{H}), 7.01(\mathrm{dd}, J=8.7 ; 1.4 \mathrm{~Hz}, 8 \mathrm{H})$ 


\section{Supplementary Figures}
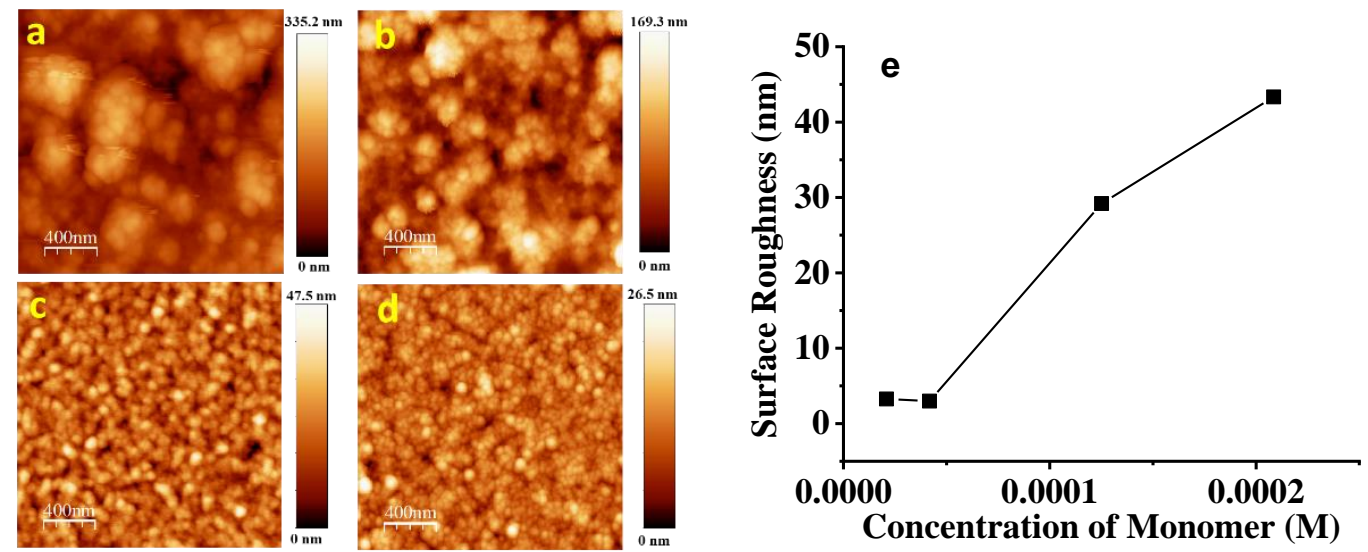

Figure S1: Surface roughness at various synthesis conditions. A-D) AFM images of films synthesized using different monomer concentrations $\left(C=2.1 * 10^{-4} \mathrm{M}\right.$ in $\mathrm{A}, 1.3 * 10^{-4} \mathrm{M}$ in $\mathrm{B}, 2.1 * 10^{-5} \mathrm{M}$ in $\mathrm{C}$, and $4.2 * 10^{-5} \mathrm{M}$ in $\left.\mathrm{D}\right)$. The concentration used throughout this study is the one in C. E) Surface roughness of the films as a function of monomer concentration when synthesizing.

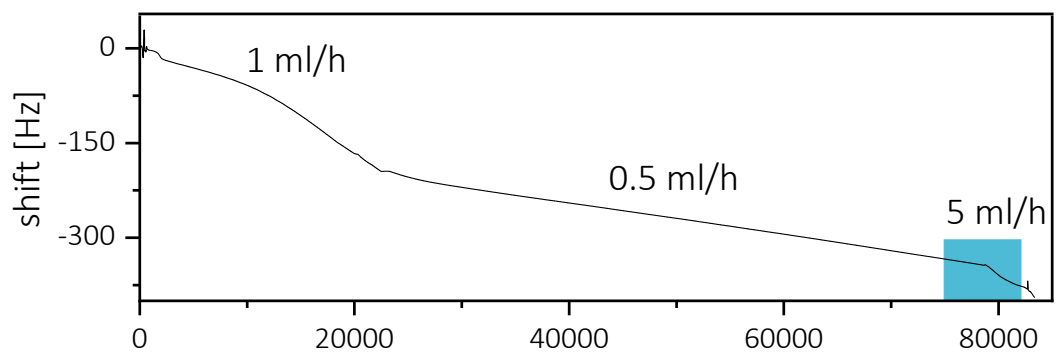

time $[\mathrm{s}]$

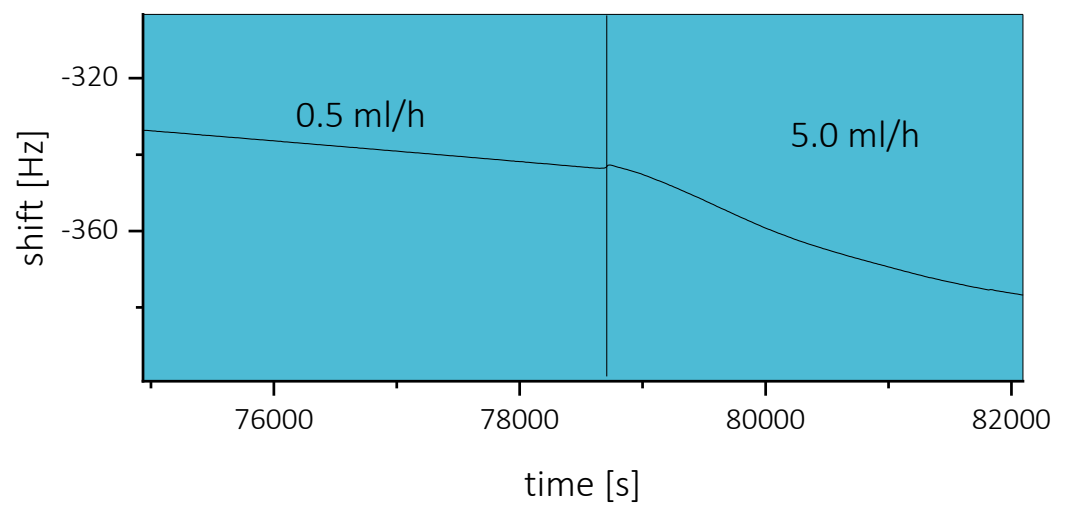

Figure S2: Dependence of the dissipation ratel film grows by the flow speed. During this experiment the flow speed was changed several times which caused a change in the slope of the frequency shift. This demonstrates that the frequency shift/reaction rate depends on the flow speed. This observation indicates that the film growing process is diffusion limited. 


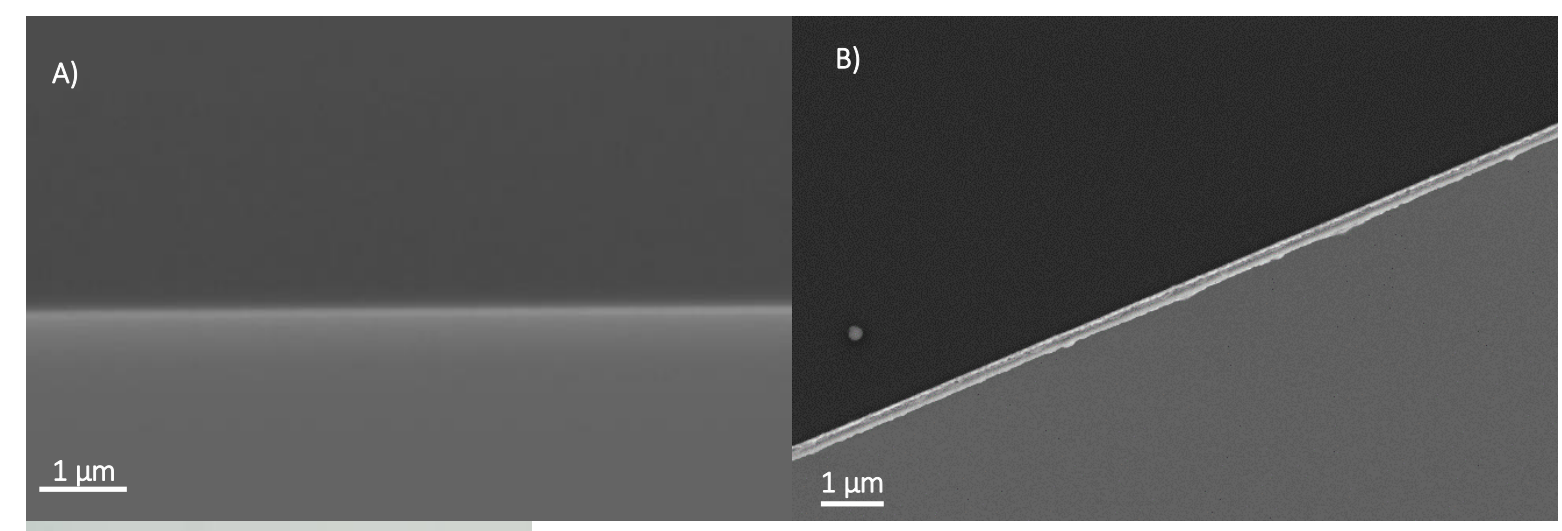

C)

$\underline{20 \mu \mathrm{m}}$
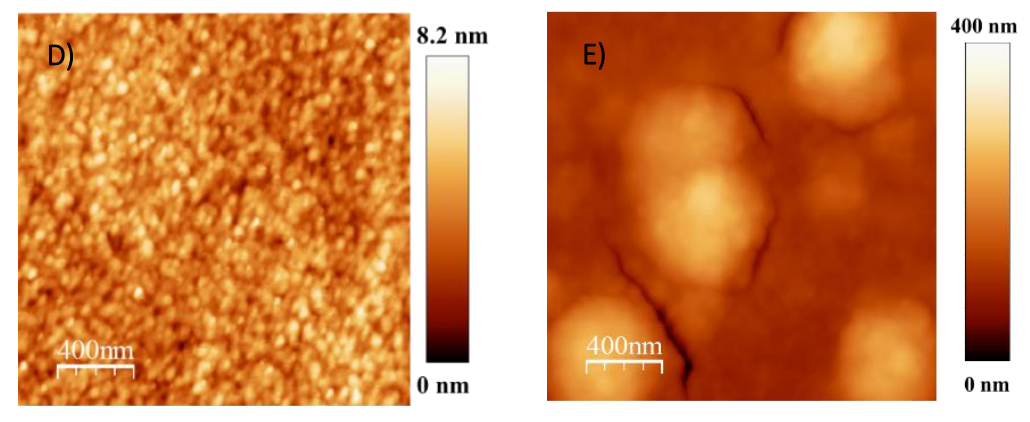

Figure S3: Additional SEM, optical microscope and AFM images of a clean gold surface. A) SEM picture of the cross section of a clean gold QCM chip. B) SEM picture with $24 \mathrm{k}$ times magnification of the film cross section, showing the existence of a smooth film. C) Image of the surface with 50 times magnification. D) AFM image of a clean gold surface. D) AFM image of a film made without having a SAM layer on the gold surface (but otherwise using the same conditions as in Figure S1c). 

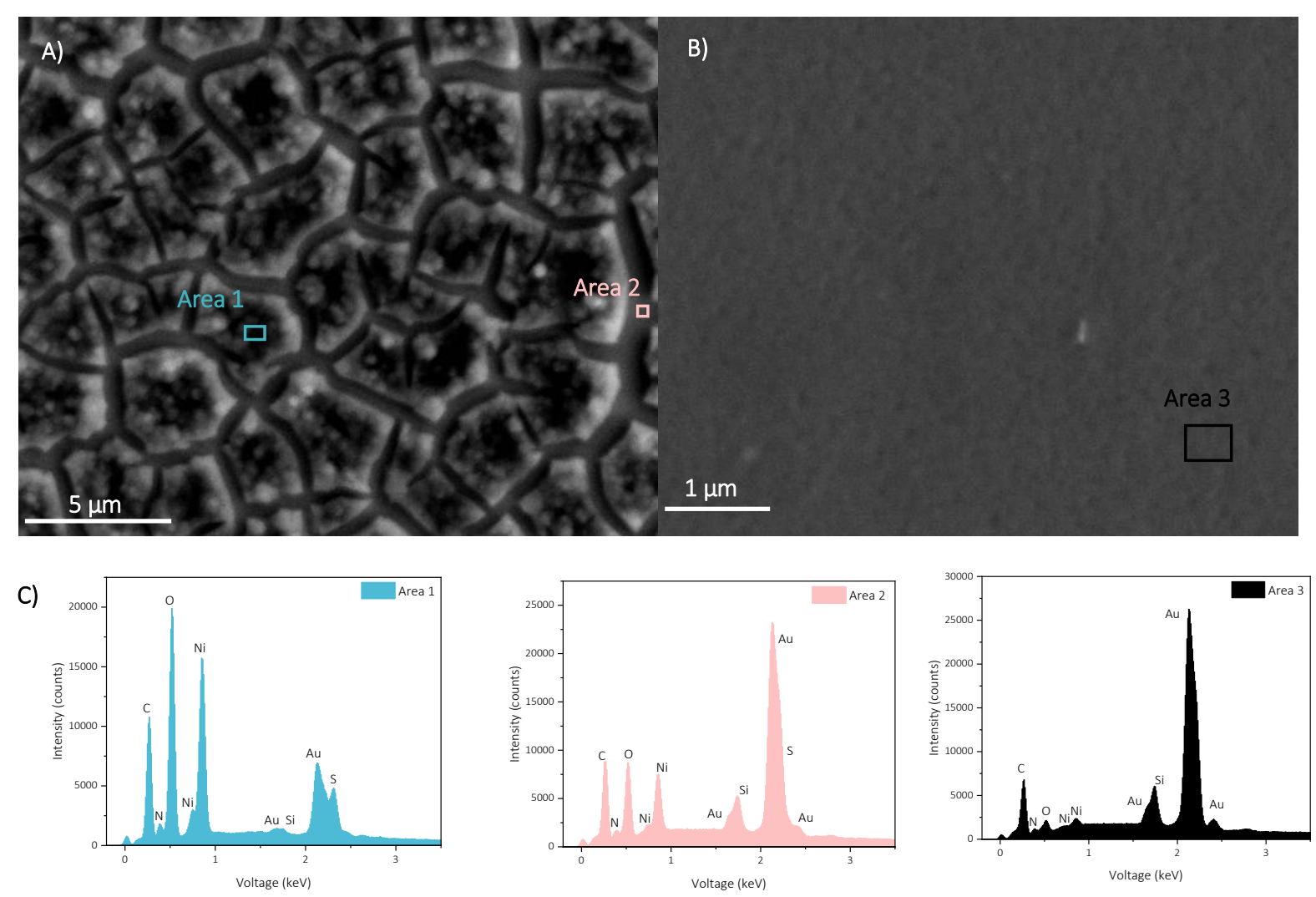

Figure S4: SEM pictures and EDX analysis from the top. SEM pictures from the top of films made using a non-brominated (A) and a brominated SAM (B) on the Au substrates. The EDX analysis of indicated areas of analysis are shown in $(C)$. The main elements which are in one of the plateaus (area 1) are carbon, oxygen (most likely solvent), nickel (from $\left.\mathrm{Ni}(\mathrm{COD})_{2}\right)$, gold (chip surface) and sulfur (SAM), whereas the canyons (area 2) show the same elements but a much higher ratio of gold and sulfur. The existence of $C, O$ and $\mathrm{Ni}$ in this area is motivated by the operating principle of an SEM. It is not possible to avoid completely the excitation of neighbor regions close to the beam (plateaus). Area 3 shows instead a smooth framework film, where mainly gold, carbon and silicon are present. 


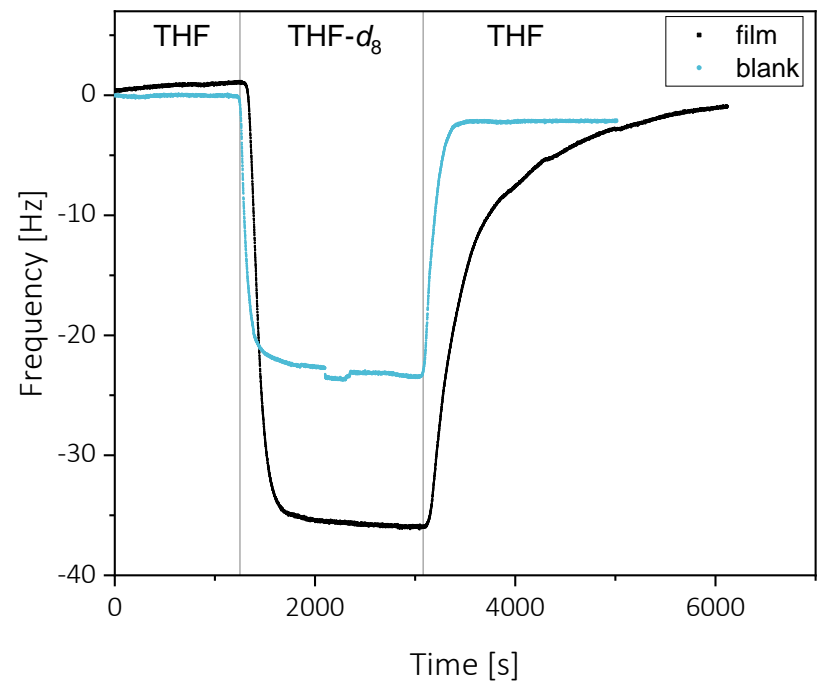

Figure S5: The solvent influence on the frequency shift. Because THF-d8 is heavier than normal THF, when changing in between these two solvents, a drop in the resonance frequency of the QCM sensor will occur if THF is exchanged to THF-d8. To verify that the observed change in the resonance frequency origins from a solvent exchange in the pores and not only from free solvent molecules flowing over the sensor, we compared the absolute value for the frequency shift for a clean gold surface (here in turquoise) and one which contains a film (black). The frequency drop is bigger for the film than for the blank substrate. This indicates that caged solvent molecules influence the frequency shift. The calculated deposited mass on the sensor, thus origins not only from the film but also from solvent molecules.

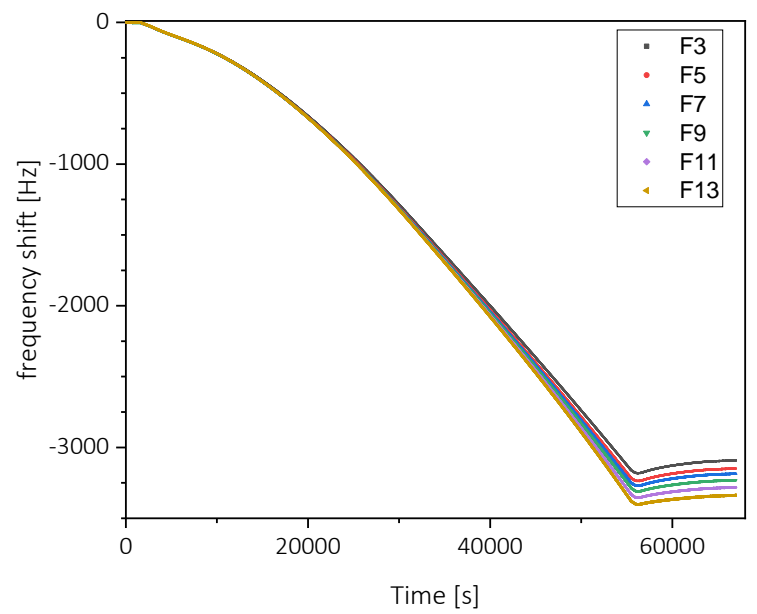

Figure S6: Plot of the different overtones F3-F13 during the experiment. Usually the $11^{\text {th }}$ overtone was used for the correlation between frequency shift and thickness of the film. 

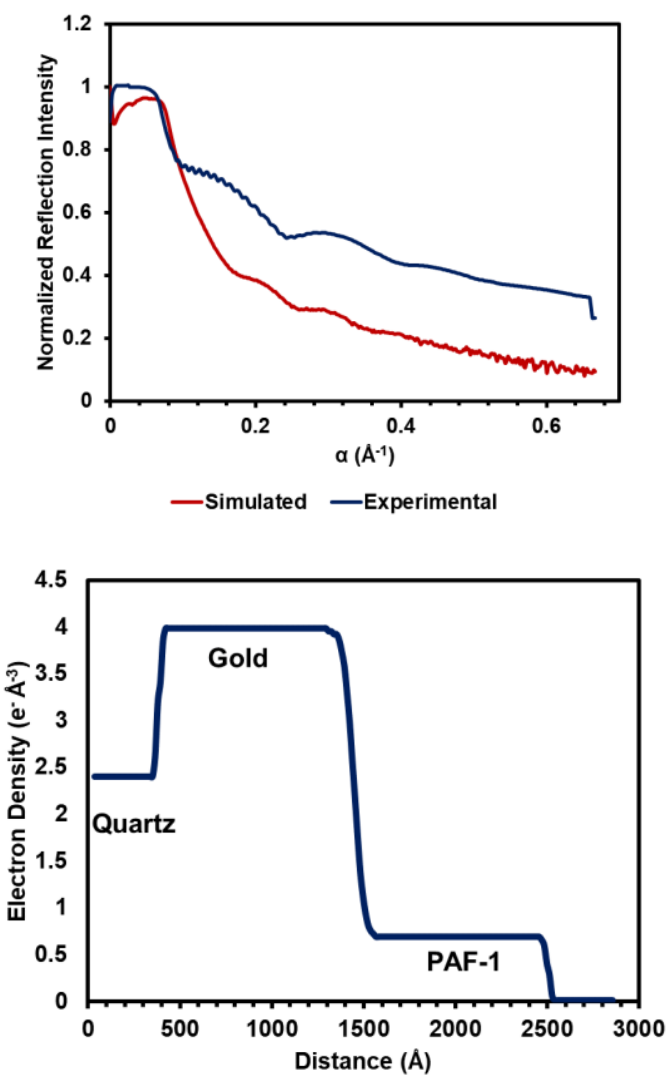

Figure S7: X-ray reflectivity of PAF-1 films on QCM wafers. (Top) Raw X-ray reflectivity intensities (simulated and experimental). (Bottom) simulated electron density profile for the substrate arrangement highlighting a low-electron density material as the top layer.

A

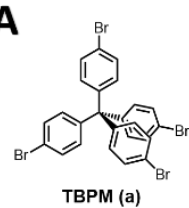

TBPM (a)

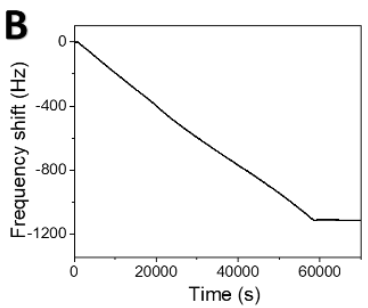

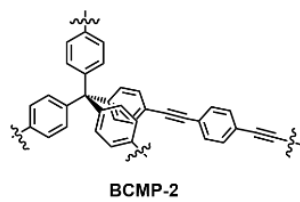

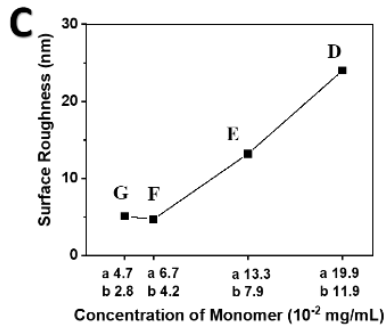

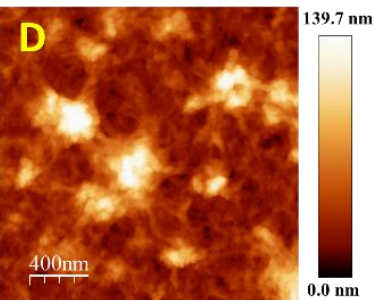
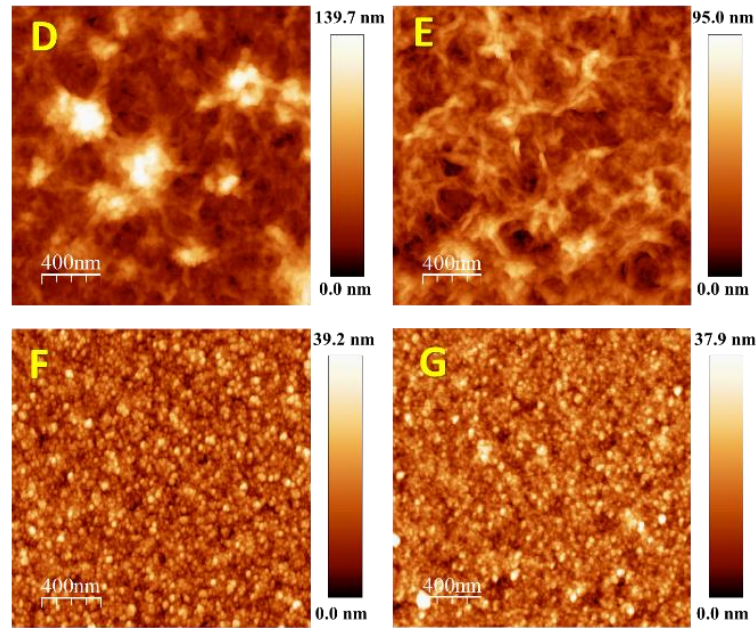

Figure S8: A Sonogashira coupling based PAF film. A) The two monomers used to build up the PAF as well as a unit of the coupled product. B) Observed frequency shift as a function of reaction time, when building up the PAF film in a QCM flow cell. C) Surface roughness as measured with AFM a function of concentration of the monomers when synthesizing the film. The concentrations are labeled D to G, which indicate respective AFM image. 


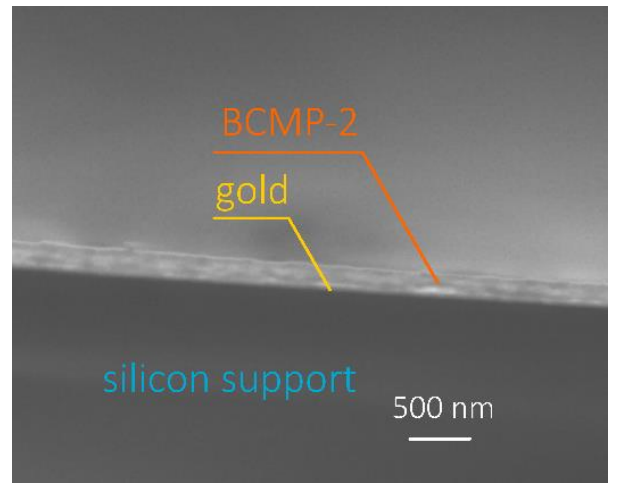

Figure S9: SEM image of the cross section of the BCMP-2 film. The images was taken using 14,000 times magnification and shows the existence of $a \sim 100 \mathrm{~nm}$ thick smooth film. 


\section{References}

1 Wang, X., Lu, S.-m., Li, J., Liu, Y. \& Li, C. Conjugated microporous polymers with chiral BINAP ligand built-in as efficient catalysts for asymmetric hydrogenation. Catal. Sci.

Technol. 5, 2585-2589 (2015).

2 Erb, R. A. Wettability of gold. The Journal of Physical Chemistry 72, 2412-2417 (1968).

3 Vignaud, G. \& Gibaud, A. REFLEX: a program for the analysis of specular X-ray and neutron reflectivity data. J. Appl. Crystallogr. 52, 201-213 (2019). 